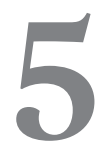

\title{
Experiencing relational depth in therapy: what we know so far
}

\section{Mick Cooper}

The aim of this chapter is to draw together findings from recent studies on the experience of relational depth, including those described earlier in this book, to give a state-of-the-art review of what the research currently tells us about moments of deep connection: its prevalence, nature, consequences and antecedents.

Before reviewing this literature, however, there is an important preliminary question to ask: Can research, and particularly quantitative, number-based inquiry, ever tell us anything meaningful about relational depth? Surely, it could be argued, relational depth is such a subtle, holistic and complex phenomenon - something that individuals find so 'hard to put into words' that to try and analyse and present it in empirical terms would be to undermine the very essence of what it is. This viewpoint is captured by a humanistic trainee interviewed by Connelly (2009), who said:

I'm quite happy for it [relational depth] to be elusive ... There's a fear of, you know - it's like kind of butterfly catching, isn't it? - there's a fear of catching something very beautiful and trying to define what it is. And then, and in that process, losing what it is.

Moreover, it could be argued that the very process of 'scientifically' measuring, quantifying and empirically examining phenomenon is antithetical to a worldview based around relational depth, with its emphasis on I-Thou relating (Buber, 1947), as opposed to I-It reductionism and objectification.

No doubt there is much validity to this argument. However, there are also ways in which an understanding of the world based around relational encounter can be seen as pointing towards the value of research inquiry. First, 
relational depth is about openness and fluidity - a willingness to move beyond fixed, sedimented assumptions - and research evidence can be a powerful means of challenging our own beliefs and expectations, and engaging more fully with what is actually 'out there' in the world (see Cooper, 2010a). As Carl Rogers (1986, cited in Cain, 2010, p. 42), for example, wrote in relation to person-centred practice:

There is only one way in which a person-centred approach can avoid becoming narrow, dogmatic and restrictive. That is through studies - simultaneously hardheaded and tender-minded - which open new vistas, bring new insights, challenge our hypotheses, enrich our theory, expand our knowledge, and involve us more deeply in an understanding of the phenomena of human change.

Second, relational depth is about moving away from all-or-nothing thinking, to an appreciation of the intricacies and complexities of any person or phenomenon. So, although research evidence may not give us definitive answers to questions about relational depth, it can be one very valuable means of edging forward in our understanding: something that should no more be discounted than it should be revered as a 'royal road' to the 'truth'.

In the review that follows, I have focused only on research that explicitly examines the concept of 'relational depth', as developed by Mearns (1997, 2003) and Mearns and Cooper (2005), and as defined in the Introduction. As discussed in the Introduction (this volume), there are several closely related concepts but, as far as we are aware, little empirical research has been undertaken in these fields. As most of the contemporary research also focuses on relational depth as a moment of experiencing (see Introduction, this volume), this is the focus of the review.

\section{Do therapists experience relational depth with their clients?}

For relational depth to be a meaningful therapeutic construct, it must be something that is actually present, at least to some extent, within the therapeutic relationship. An important initial question, therefore, is: Do therapists actually experience relational depth with their clients and, if so, are there any particular kinds of therapists who are more likely to experience it than others?

Probably the best evidence in relation this question comes from an online survey conducted by Leung (2008). His respondents were 140 therapists from a variety of orientations (although primarily humanistic), and he found that almost 98 per cent reported at least one experience of relational depth with a client. Moments of relational depth in therapy could also be identified by eight out of eight experienced person-centred therapists in a qualitative interview study (Cooper, 2005), and nine out of ten primarily person-centred/ 
humanistic therapists who worked with clients with learning disabilities (see Chapter 5, this volume).

In terms of prevalence, therapists in the Leung survey were asked to rate on a 7 -point scale how frequently they had experienced moments of relational depth with their clients $(1=$ not at all, $7=$ all the time). The average rating was around the midpoint of the scale, 4.06.

These data suggest that a large majority of therapists have experienced moments of profound connection with a client, and at a moderate level of frequency. However, there are three reasons why the actual percentage of therapists who have experienced relational depth may be somewhat lower than this suggests:

1. Respondents to these studies were self-selecting, such that therapists with a greater interest in the therapeutic relationship - and potentially more likely to experience relational depth - may have been more likely to take part.

2. Participants may have wanted to present themselves, particularly in the interview studies, in a positive light, hence overstating the extent to which they had experienced moments of deep encounter.

3. The majority of participants in these studies were of a person-centred or humanistic orientation - therapeutic approaches that place strong emphasis on the relationship - such that it is not clear whether these figures would generalize out to less relationally oriented therapies.

With respect to the last of these points, however, Leung (2008) found no significant differences in the extent to which humanistic, psychodynamic and other (mainly cognitive behavioural therapy) practitioners reported experiencing relational depth. Nevertheless, with respect to differences across professional groups, Morris (2009) did find that psychologists (clinical and counselling) working in the NHS were less likely to recognize such experiences of encounter in their work, with just three out of six interviewees (50 per cent) identifying moments of relational depth.

In terms of other individual variables, Leung (2008) found no significant differences between male and female therapists. However, therapists with more years of practice did report a greater frequency of relationally deep encounters.

In summary, then, research suggests that a large proportion of therapists, perhaps most, have experienced moments of profound contact with their clients. Evidence is strongest for practitioners of a person-centred or humanistic orientation, and with some indications that more experienced practitioners are most likely to experience relational depth.

\section{Do clients experience relational depth with their therapists?}

Early literature and research on relational depth (for example Cooper, 2005; 
Mearns and Cooper, 2005), as above, tended to focus on relational depth as experienced by therapists. Perhaps this was on the assumption that, as a mutual, bidirectional experience, if therapists were experiencing relational depth, clients would be too. But this assumption is by no means a given. Indeed, from much of the psychotherapy research, it is evident just how different clients' and therapists' experiences of the same relational encounters can be (see, for example, Cooper, 2008, p. 2). And given that, for relational depth to be healing, it must be actually experienced by the client, a critical question to ask is whether clients, as well as therapists, do also experience moments of profound connection in therapy.

Again, some of the best evidence for this comes from Leung's (2008) online survey. Of 119 participants who responded as clients, Leung found that 78.2 per cent could identify a moment of relational depth. This is significantly less than the proportion of therapists identifying moments of profound encounter with their clients, but still a substantial proportion of respondents. Knox, as discussed in Chapter 2 (this volume), also found a relatively high proportion of clients identifying moments of relational depth with their therapists; and all three of the clients interviewed by Omielan (2009) in his series of narrative case studies also described moments of profound connection.

In terms of frequency of experiencing moments of relational depth, clients in the Leung (2008) survey gave a mean rating of 3.87 on the 7 -point scale ( $1=$ not at all, $7=$ all the time): again towards the midpoint of the scale and not significantly different from therapists.

McMillan and McLeod (2006, p. 289), however, obtained a somewhat different picture from their qualitative study of ten clients' experiences of the therapeutic relationship. Here, specific moments of intense closeness were found to be 'relatively rare'. In attempting to reconcile these findings with those of Leung (2008) and Knox (2011), it is important to note that the latter studies asked participants directly about their experiences of relational depth, while McMillan and McLeod (2006) inquired more broadly about the therapeutic relationship. Hence, with McMillan and McLeod (2006), some experiences of relational depth may have been overlooked; while in the former studies, participants may have felt under some pressure to identify specific moments.

In each of these studies, there are also the problems, again, of self-selected participants, impression management concerns, and many of the clients also being therapists, meaning that the actual percentage of clients who have experienced moments of profound connection may be considerably less than the 78.2 per cent identified by Leung. At the same time, however, the fact that clients were significantly less likely to have experienced relational depth than therapists may be attributable to the fact that, while clients may only have worked with one or two therapists, therapists may have worked with tens or even hundreds of clients - hence having much more opportunity to experience relational depth. In this respect, it is by no means clear whether thera- 
pists or clients, over an equivalent number of relationships and/or period of time, would be most likely to experience a profound depth of connection.

In terms of which kinds of clients may be most likely to experience relational depth, Leung (2008) found just one significant difference: clients of humanistic therapists were significantly more likely to report this experience than clients of psychodynamic therapists.

In summary, there is evidence that at least some clients have experienced moments of profound connection with their therapists, although it is unclear how common this experience is across a typical client population.

\section{Do clients and therapists experience relational depth at the same time?}

There is evidence to suggest, then, that both therapist and client in a therapeutic relationship may experience moments of profound connection to the other. But will they experience it at the same time, that is, 'synchronously'? In other words, if a therapist is feeling deeply connected to their client, can they assume that their client is also experiencing a profound sense of connection at that moment, or could it be that the client is experiencing something altogether different?

In an attempt to answer this question, an 'analogue' study was set up, that is, a study not using bona fide clients, in which pairs of practising or trainee counsellors were asked to conduct 'counselling' sessions of 20 minutes in length (Cooper, 2010b). 'Clients' were asked to speak about anything of concern, and therapists were asked to respond as they normally would do to their clients. The one difference was that, at each minute, participants were asked to rate how deeply connected they felt to their partner using a zero to ten scale $(0=$ not at all connected, $10=$ deeply connected $)$. This, then, gave an opportunity to see how closely therapists' and clients' ratings of connection to the other would match over time.

Results of this study suggested that the degree of synchrony between therapists' and clients' perceptions of connection is actually relatively high, with a mean correlation of around 0.67 across 80 pairs (a correlation of 0 means no matching at all, a correlation of 1 means exact matching). This translates into an average overlap in perceptions of around 45 per cent. Figure 5.1 illustrates ratings from one therapist-client pair of about this magnitude. As can be seen here, while there are some moments in which some disparity exists (for example minute 6 , in which the client experiences a reduction in depth of connection but not the therapist), in general, the degree of matching is fairly marked - even above and beyond a general deepening of connection as the session progresses; for instance minute 15 , in which both participants experience a reduction in depth of connection, and then a return to greater engagement at minute 17 . 


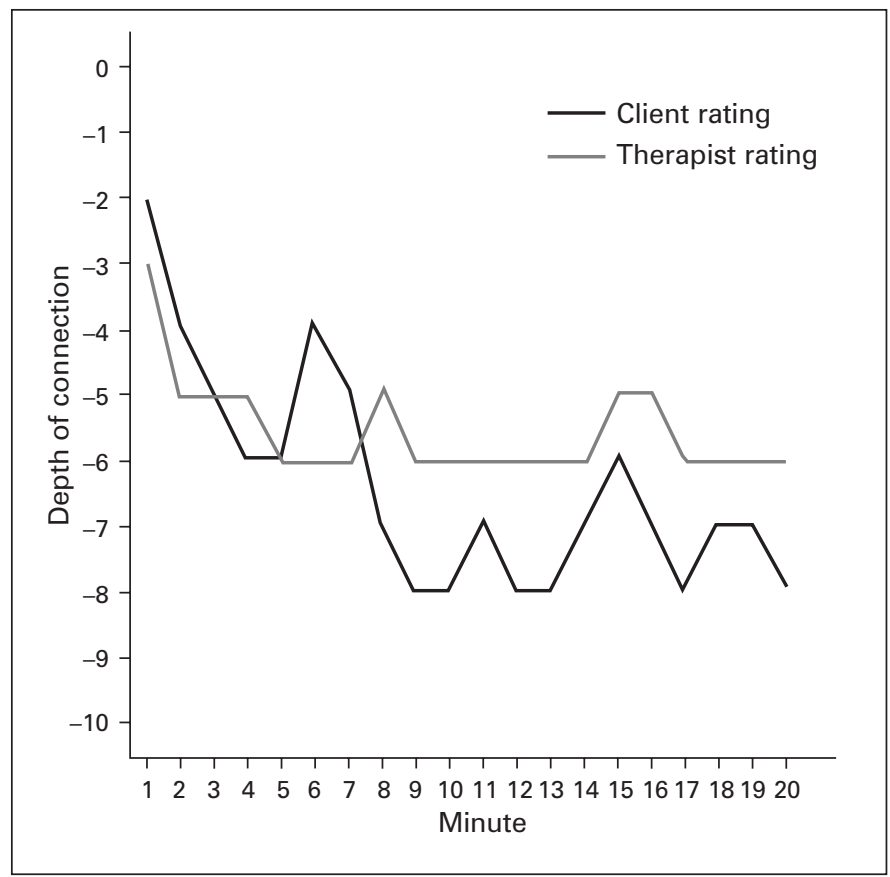

Figure 5.1 Client and therapist ratings of depth of connection Note: Lower scores indicate greater depth of connection.

This research has several limitations. First, participants were all trainee and practising therapists, such that the 'clients' may have been much more able to tune in to their therapists than most everyday users of therapy. The sessions were also artificial, relatively short, and there was no build-up of a long-term therapeutic relationship. In this respect, the study was much more about feelings of connection than profound moments of relational depth. Of course, asking a person to rate their degree of connection to another person in an actual session is also likely to have made it more difficult for them to relate, although a single case study that invited participants to rate their levels of connection after the session also indicated moderate levels of synchrony (Frzina, 2012). Finally, it is quite possible that the relatively high correlation between the two sets of ratings was due to both client and therapist experiencing a general deepening of relating over time, rather than any specific synchrony in experiencing. Nevertheless, two people's ratings of how connected they feel to each other do seem to show some similarity, suggesting that the experience of relational depth may, at least for some of the time, be a genuinely two-person, mutually experienced phenomenon. This also means that therapists can be relatively assured that, if they are feeling deeply 
connected to their client, there is a good chance (but not a certainty) that the client will be experiencing this as well.

\section{What is it like to experience relational depth?}

How does it feel to encounter another at a level of profound depth? More importantly, perhaps, is there some shared, specific experience that a wide range of individuals can recognize, or is relational depth a much more diffuse phenomenon, varying markedly from individual to individual? This was one of the first empirical questions to be asked (Cooper, 2005), and my initial study of person-centred therapists' experiences of relational depth has been followed by interview studies of psychologists' experiences (Morris, 2009), clients' experiences (Knox, 2008, 2011, Chapter 2, this volume; McMillan and McLeod, 2006), trainee therapists' experiences (Connelly, 2009), and the experiences of therapists working with clients with learning disabilities (Macleod, 2009, Chapter 3, this volume). There has also been a 'mixed methods', that is, combining quantitative and qualitative inquiry, study of clients' and therapists' experiences of relational depth, which included an online survey (Wiggins, 2007, Chapter 4, this volume).

In my initial study (Cooper, 2005), the experience of relational depth was understood in terms of three overarching domains: 'self-experiences', 'experiences of the other' and 'experiences of the relationship'. Knox (2008, 2011) and Wiggins (2007, Chapter 4, this volume) adopted this structure but added a fourth domain, 'experience of the moment itself or atmosphere'; and this four-domain structure was subsequently used by Macleod (2009, Chapter 3, this volume). Connelly (2009) developed a different set of four domains: 'physical', 'emotional', 'spiritual' and 'silence'; as did Morris (2009) with her three domains of 'empathic attunement', 'relational connectedness' and 'use of self'; but these two structures can be relatively easily subsumed into the fourdomain structure established by Knox and Wiggins.

\section{Self-experiences}

Self-experiences, the first of these four domains, are those things that participants reported experiencing 'in' themselves at times of relational depth. Across the studies (Connelly, 2009; Cooper, 2005; Knox, 2008, 2011; Macleod, 2009; Morris, 2009; Wiggins, 2007; Chapters 2-4, this volume), one of the most common features here was a sense of aliveness: a feeling of energy, exhilaration, empowerment and revitalization, often with a heightened awareness and a greater perceptual clarity. Participants also described feeling spontaneous, free and in-the-moment, with a sense of being very authentic, real, congruent and open at these times - a wholeness or fullness of being. 
Frequently, participants described very physical sensations during moments of relational depth; for example electrifying or tingly feelings, and a level of emotional intensity. Paradoxically, however, participants also described feelings of slowing down at moments of relational depth - a sense of calm, peacefulness, relaxation, safety and stillness. They also talked about being immersed in the moment, absorbed, and free from distractions, with some participants likening it to an altered state of consciousness.

Moments of deep connection were generally associated with feelings of satisfaction, happiness, wellbeing and warmth, with a sense of self-worth, selfacceptance and specialness.

\section{Experiences of other}

At these times of relational depth, others were experienced as very genuine: real, human and present - coming from the 'core' of their being (Connelly, 2009; Cooper, 2005; Knox, 2008, 2011; Macleod, 2009; McMillan and McLeod, 2006; Morris, 2009; Wiggins, 2007; Chapters 2-4, this volume).

\section{Experiences of the relationship}

Participants described powerful feelings of connection, closeness and intimacy with the other at these moments of deep connection (Connelly, 2009; Cooper, 2005; Knox, 2008, 2011; Macleod, 2009; Morris, 2009; Wiggins, 2007; Chapters 2-4, this volume) - a 'heart-to-heart' meeting - often taking place without words. A deep sense of trust was experienced, with some participants also describing feelings of love. At these times of connection, high levels of mutuality were also often felt (see Chapter 14, this volume): a sense of equality, partnership, or of being on a journey together, with a co-acceptance, co-openness and co-reflectiveness. Some participants also described experiencing a bidirectional flow: 'like electricity flowing one from the other' (Macleod, 2009, p. 42); and others described a mutually enhancing reciprocity: not just that they knew the other, but that they knew the other knew that they knew. In a few instances, participants also described feelings of union, fusing or blending: an interlinking or blurring of boundaries.

\section{Experiences of the moment itself}

In some of the studies (Connelly, 2009; Cooper, 2005; Knox, 2008, 2011; Macleod, 2009; Wiggins, 2007; Chapters 2-4, this volume), participants described aspects of the experience of relational depth that were less to do with specific intra- or interpersonal experience and more to do with the 
moment itself, or with the particular atmosphere surrounding the moment. For instance, participants described experiencing these moments of relational depth as unique, rare or strange: something that was surprising, unexpected and difficult to put into words. They also described them as meaningful or significant. Another common feeling during these moments was of being on a different dimension or level, or a strong spiritual or mystical connection. Changes in the perception of time were also commonly described - that it was standing still or being distorted in some way.

\section{Unidimensionality}

Although this four-domain structure is a useful means of conceptualizing relational depth, research suggests that the experience of relational depth is a relatively holistic, integrated phenomenon. Indeed, a statistical analysis conducted by Wiggins (2007) suggests that many elements of each of these domains overlap with elements of other domains. In a subsequent analysis, Wiggins found that her Relational Depth Inventory (RDI, a measure designed to assess the depth of particular moments in therapy; see Chapter 4, this volume) was 'pretty much one-dimensional' (personal communication, 2011), suggesting that the principal features associated with an experiencing of relational depth do seem to exist concurrently.

\section{Variations across experiences}

In general, a remarkable degree of consistency existed in participants' descriptions of the experiences of relational depth - both across participants in the same study and across studies (Connelly, 2009; Cooper, 2005; Knox, 2008, 2011; Macleod, 2009; Wiggins, 2007; Chapters 2-4, this volume).

However, some significant differences have emerged in how clients and therapists described their experiences of relational depth, consistent with their specific roles. For instance, in terms of self-experiences, therapists were particularly likely to describe feelings of respect and empathy for others (Wiggins et al., 2012), heightened empathy and focus, with an enhanced acceptance of the other, and a feeling of being privileged to engage with their clients at this level of depth (Cooper, 2005; Macleod, 2009; Morris, 2009). By contrast, at times of relational depth, clients were more likely to describe feelings of being known, understood, cared for and accepted; as well as feeling vulnerable (Knox, 2008, 2011; Wiggins et al., 2012) and scared (Wiggins et al., 2012) and delving deeply into themselves (Knox, 2008, 2011, Chapter 2, this volume). Consistent with this, therapists were more likely to perceive their clients as vulnerable and opening up at these times (Cooper, 2005; Macleod, 2009; Morris, 2009); while clients were more likely to perceive their therapists as empathic, warm, inviting and creating opportunities, with a sense of 
reliability, trustworthiness or solidity; and offering something 'over and above' the core conditions (see below) (Knox, 2008, 2011, Chapter 2, this volume).

These differences between therapists' and clients' experiences of relational depth raise the question of whether it is, indeed, a mutual experience - with bidirectional feelings of empathy, understanding and openness - or whether, as McMillan and McLeod (2006, p. 289) argue, this is primarily the therapists' perception. What evidence there is so far suggests that the answer is probably somewhere in between. On the one hand, as discussed above, therapists are much more likely to experience feelings of empathy and understanding towards the other at times of relational depth than clients; and, indeed, items on 'understanding the therapist' and 'knowing the other's mind' were among the least endorsed by clients on the RDI (Wiggins, 2011a). On the other hand, and contrary to McMillan and McLeod's findings, clients have specifically described feelings of mutuality with their therapists at times of relational depth (Knox, 2008, 2011, Chapter 2, this volume) and, perhaps surprisingly, this is most marked in non-therapist clients. One client stated, for instance:

There are times when we two, very open ... damaged individuals. She [the therapist] opens up her damage to me and I open my damage to her ... And when we do connect, and when [name of therapist] affirms me, what I need that, I am actually contributing to her life, and I know she's contributing to mine. (Knox, 2011, p. 210)

\section{Summary}

Research into the experience of relational depth paints a vivid picture of what it is like to meet another human being at this level of profound connectedness. It is, to summarize:

A sense of connectedness and flow with another person that is so powerful that it can feel quite magical. At these times, the person feels alive, immersed in the encounter, and truly themselves; while experiencing the other as open, genuine and valuing of who they are. (Cooper, 2009)

The striking commonalities in how this experience is described - both across participants within the same study and across studies - suggest that the phenomenon of relational depth 'is a real and distinctive occurrence' (Cooper, 2005, p. 93). At the same time, however, the evidence does suggest that there are certain asymmetries in how moments of relational depth are experienced by therapists and clients, with the former more likely to experience feelings of empathy and acceptance towards the other, and the latter more likely to experience feelings of vulnerability and self-inquiry. Whether this is a fundamental challenge to the assumption of mutuality in relational depth, however, or simply a difference of focus and content, remains to be seen. 


\section{What is the relationship between relational depth and therapeutic outcomes?}

A key assumption behind the concept of relational depth (see, for example, Mearns and Cooper, 2005), indeed, its very raison d'être, is that deeper levels of relating lead to 'better' outcomes in counselling and psychotherapy. But is there actually any evidence that this is the case?

Some of the strongest evidence to support this hypothesis comes from a recent study by Wiggins (2011b, Chapter 4, this volume). Using her RDI, Wiggins invited clients to identify a particular helpful moment or event in therapy, and then to rate how accurately 24 items associated with relational depth fitted with this experience. Wiggins then looked at whether relational depth was predictive of positive therapeutic outcome, and found that it was, with depth of relating accounting for a striking 10-30 per cent of the overall outcomes. In other words, the more that clients experienced relational depth at a particular helpful moment in therapy, the more they improved.

In addition, research by Murphy (2010, Chapter 14, this volume) suggests that the association between clients' rating of the therapist core conditions and outcome is enhanced when mutuality exists in therapists' and clients' perceptions of the therapeutic relationship.

More subjective evidence come from Leung (2008), who asked participants to rate, on a 7-point scale, how important they thought moments of relational depth were for personal change and for the outcomes of therapy ( $1=$ not at all, $7=$ extremely). On average, therapists gave it a rating of 5.69 and clients gave it a rating of 5.78, indicating that these moments were seen as being very important for therapeutic change, with no significant difference between therapists' and clients' ratings. Participants were also asked to rate, using a similar 7-point scale, the extent to which they thought these moments of relational depth had an enduring impact. Both therapists and clients gave this a mean rating of 5.87, again suggesting that moments of relational depth were seen as being strongly therapeutic. A majority of clients in Knox's (2008, 2011, Chapter 2, this volume) interview studies also felt that moments of relational depth had had a significant positive impact, both immediately and in the longer term; as did clients interviewed by Omielan (2009).

In terms of the specific effects that a meeting at relational depth might have, participants in Knox's (2008, 2011, Chapter 2, this volume) research identified two immediate impacts: a general experience of the moments as facilitative, healing and changing; and a positive effect on the therapeutic process itself. Participants in these studies also described the long-term effects of these moments: an increased sense of connection to their own selves, feeling more powerful, feeling better, and improved relationships with others.

In summary, new research by Wiggins (2011b) and Murphy (2010) suggests that the depth of relating and mutuality between therapists and clients at particular moments in therapy is strongly predictive of positive therapeutic outcomes. However, these studies are in need of replication, while 
it is important to emphasize that they do not necessarily establish causality: it may be, for instance, that a third factor, such as clients' levels of motivation, determines both the depth of relating and outcomes. More qualitative evidence, however, does support the view that moments of in-depth relating have a direct positive effect.

\section{What facilitates a meeting at relational depth?}

A meeting at relational depth, as Mearns and Cooper (2005) argue, is not something that therapists can make happen. But are there ways in which the likelihood of such a depth of encounter can be enhanced? Given, as indicated above, that such meetings may have important therapeutic benefits, this may be a key question to ask. Research on facilitative factors, as well as inhibiting ones, is still in its infancy (Knox, 2011; Knox and Cooper, 2010, 2011; Macleod, 2009; see Chapters 2 and 3, this volume), yet, already, some of the most fascinating findings in the field are beginning to emerge here.

\section{Therapist factors}

In looking at the therapist factors that were associated with the emergence of moments of relational depth, Knox (2011, Chapter 2, this volume; Knox and Cooper, 2010) identified two main domains: who therapists were, and what therapists did. In terms of the first of these categories, 'therapists' personal characteristics', clients described the therapists who they had related at depth with as warmth, empathic and courteous. In addition, they had experienced these therapists as psychologically sound: strong, comfortable with their own selves, and willing to relate at depth. Perhaps most interestingly, though, clients also felt that these moments of relational depth had taken place with therapists who were, in some way, similar or matching to themselves, for example with similar beliefs or perceived lifestyles. The clients also described these therapists as the 'right' sort of person, with some, particularly the nontherapists, likening them to the mother or father they had never had. This last finding is consistent with research by McMillan and MacLeod (2006, p. 285), who found that many participants described their therapist in deeply facilitative relationship as 'like a good or ideal mother'.

In terms of what a therapist who they had related at depth with had done, Knox (2011, Chapter 2, this volume; Knox and Cooper, 2010) found that clients emphasized their patience, their ability to create a welcoming and safe atmosphere, and to act in a way that was trustworthy, reliable and professional. Being accepting, open and understanding were also important features of these therapists. However, two further, particularly interesting factors also emerged. First, clients talked about the way these therapists had been really real and human: just genuine, themselves and not faking things or putting 
themselves on a professional pedestal. Second, and very much in line with findings from McMillan and MacLeod (2006, p. 285), they describe these therapists as offering something 'over and above' what they would expect from a professional relationship: a genuine, very real commitment and care. This experience of feeling deeply cared for and nurtured in relationships in which relational depth was experienced was also identified by Omielan (2009). Interestingly, too, the clients emphasized how the therapists who they had met at depth felt really on their side: not just a neutral, nonjudgemental presence, but someone who actively prized them, and saw things in their way. They also talked about the therapist's encouragement and pride in them, and holding the hope for their future.

\section{Client factors}

Perhaps the most interesting finding to emerge from Knox's (2011, Chapter 2, this volume; Knox and Cooper, 2010, 2011) interview studies with clients, however, is that the key precursors of a meeting at relational depth may be less to do with therapists, and more to do with the clients themselves. Clients, in these studies, did describe how challenges from the therapist, or changes in how they experienced the therapist or the relationship, preceded moments of relational depth. But they also talked extensively about the importance of their own readiness and desire to engage at depth, and how that had been an essential precursor to the depth of meeting. In addition, while clients talked about heightened levels of emotion prior to the moment of relational depth, they also described it as something they had proactively entered into: a deliberate choice to take the risk and open up.

\section{Summary}

While therapists cannot make moments of relational depth happen, the research suggests that they may be able to facilitate their emergence through communicating a genuine care and commitment to clients, as well as creating a safe, professional and warm therapeutic environment. Ultimately, however, it may be that clients, rather than therapists, are the principal factor in determining whether or not meetings at depth will take place, and more evidence is needed on what it is that clients do to make this happen.

\section{What factors inhibit a meeting at relational depth?}

Unsurprisingly perhaps, clients' perceptions of the qualities of therapeutic relationships in which relational depth was not experienced was the near reverse of those in which it was (Knox, 2011, Chapter 2, this volume; Knox 
and Cooper, 2010). Therapists were experienced as cold and distant, uncaring, and failing to understand the client or invite them into deeper levels of relating. In some cases, they were also experienced as too different from the client, and using a counselling style that the client did not feel suited them. In addition, they tended to perceive these therapists as inexperienced and unprofessional and, in some instances, manipulative or misusing power.

Research from Connelly (2009) with trainee therapists also found that fear and anxiety were experienced as inhibiting an openness to relational depth, as were personal defences. Participants also spoke of the risk of being shamed.

\section{Conclusion}

To summarize what the research tells us so far: a majority of therapists, particularly of a person-centred and humanistic orientation, seem to have experienced moments of relational depth with their clients. At least some clients seem to have experienced these moments too, and there is some evidence to suggest that this experiencing is relatively synchronous. The experience itself can be described as a feeling of deep immersion in a connection with another human being who is experienced as very genuine and real. There is a growing body of evidence to suggest that the experiencing of moments of relational depth is associated with positive therapeutic outcomes, and it seems that therapists can facilitate the likelihood that these moments will emerge by expressing their genuine care and commitment in the therapeutic relationship. Ultimately, however, it may be that clients are the principal determinants of whether or not an encounter at relational depth takes place.

Relational depth is not something that we can, or would ever want to, pin down. It exists by virtue of its mystery, its ability to surprise and take hold of us and transform our lives in ways that we cannot predict or control. And yet, by discovering more about it, we can help to keep it central to our lives and our work: a never-ending inquiry into the heart of therapeutic relating, where the journey, and not the destination, is the ultimate goal.

\section{References}

Buber, M. (1947) Between Man and Man (trans. R.G. Smith). London: Fontana.

Cain, D.J. (2010) Person-centered Psychotherapies. Washington, DC: APA.

Connelly, A.E. (2009) Trainee therapists and relational depth. Unpublished MSc dissertation, Sherwood Psychotherapy Training Institute, Nottingham.

Cooper, M. (2005) Therapists' experiences of relational depth: A qualitative interview study. Counselling and Psychotherapy Research, 5(2), 87-95.

Cooper, M. (2008) Essential Research Findings in Counselling and Psychotherapy: The Facts are Friendly. London: Sage.

Cooper, M. (2009) Relational depth: Where we are now. Paper presented at the 2nd Relational Depth Research Conference, University of Nottingham. 
Cooper, M. (2010a) The challenge of counselling and psychotherapy research. Counselling and Psychotherapy Research, 10(3), 183-91.

Cooper, M. (2010b) Synchrony in clients' and therapists' experiences of relational depth: An analogue study. Paper presented at the COSCA 7th Counselling Research Dialogue, Sterling.

Frzina, J. (2012) A case study exploring experience of relational depth between therapist and client in a single session recorded during a skills practice. Counselling Psychology Review, 27(2), 52-62.

Hatcher, R.L. and Gillaspy, J.A. (2006) Development and validation of a revised short version of the Working Alliance Inventory. Psychotherapy Research, 16(1), 12-25.

Knox, R. (2008) Clients' experiences of relational depth in person-centred counselling. Counselling and Psychotherapy Research, 8(3), 118-24.

Knox, R. (2011) Clients' experiences of relational depth. Unpublished PhD dissertation, University of Strathclyde, Glasgow.

Knox, R. and Cooper, M. (2010) Relationship qualities that are associated with moments of relational depth: The client's perspective. Person-Centered and Experiential Psychotherapies, 9(3), 236-56.

Knox, R. and Cooper, M. (2011) A state of readiness: An exploration of the client's role in meeting at relational depth. Journal of Humanistic Psychology, 51(1), 61-81.

Leung, J. (2008) A quantitative online study exploring the factors associated with the experience and perception of relational depth. Unpublished DPsych dissertation, University of Strathclyde, Glasgow.

Macleod, E. (2009) A qualitative exploration into therapists' perceptions of reaching relational depth when counselling people with learning disabilities. Unpublished MSc dissertation, University of Strathclyde, Glasgow.

McMillan, M. and McLeod, J. (2006) Letting go: The client's experience of relational depth. Person-Centered and Experiential Psychotherapies, 5(4), 277-92.

Mearns, D. (1997) Person-Centred Counselling Training. London: Sage.

Mearns, D. (2003) Developing Person-Centred Counselling (2nd edn). London: Sage.

Mearns, D. and Cooper, M. (2005) Working at Relational Depth in Counselling and Psychotherapy. London: Sage.

Morris, G. (2009) Psychologists' experiences of relational depth: A qualitative interview study. Unpublished DPsych thesis, University of Strathclyde, Glasgow.

Murphy, D. (2010) Psychotherapy as mutual encounter: A study of therapeutic conditions. Unpublished $\mathrm{PhD}$ dissertation, Loughborough University, Loughborough.

Omielan, R. (2009) The influence of relational depth on therapeutic relationships: A narrative inquiry into client experience. Unpublished MSc dissertation, University of Bristol, Bristol.

Wiggins, S. (2007) Developing an inventory designed to assess relational depth. Unpublished MSc dissertation, University of Strathclyde, Glasgow.

Wiggins, S. (2011a) Development and validation of a measure of relational depth. Unpublished $\mathrm{PhD}$ dissertation, University of Strathclyde, Glasgow.

Wiggins, S. (2011b) Relational depth and therapeutic outcome. Paper presented at the 17th Annual BACP Research Conference, Edinburgh.

Wiggins, S., Elliott, R. and Cooper, M. (2012) The prevalence and characteristics of relational depth events in psychotherapy. Psychotherapy Research, 22(2), 139-58. 